\title{
Pituitary dysfunction induced by immune checkpoint inhibitors is associated with better overall survival in both malignant melanoma and non- small cell lung carcinoma: a prospective study
}

Tomoko Kobayashi, ${ }^{1}$ Shintaro Iwama (D , ${ }^{1}$ Yoshinori Yasuda, ${ }^{1}$ Norio Okada, ${ }^{1}$ Takayuki Okuji, ${ }^{1}$ Masaaki Ito, ${ }^{1}$ Takeshi Onoue, ${ }^{1}$ Motomitsu Goto, ${ }^{1}$ Mariko Sugiyama, ${ }^{1}$ Taku Tsunekawa, ${ }^{1}$ Hiroshi Takagi, ${ }^{1}$ Daisuke Hagiwara, ${ }^{1}$ Yoshihiro Ito, ${ }^{1,2}$ Hidetaka Suga, ${ }^{1}$ Ryoichi Banno, ${ }^{1,3}$ Kenji Yokota, ${ }^{4}$ Tetsunari Hase, ${ }^{5}$ Masahiro Morise, ${ }^{5}$ Naozumi Hashimoto, ${ }^{5}$ Masahiko Ando, ${ }^{6}$ Yasushi Fujimoto, ${ }^{7}$ Hideharu Hibi, ${ }^{8}$ Michihiko Sone, ${ }^{7}$ Yuichi Ando, ${ }^{9}$ Masashi Akiyama, ${ }^{4}$ Yoshinori Hasegawa, ${ }^{5}$ Hiroshi Arima ${ }^{1}$

To cite: Kobayashi T, Iwama S, Yasuda Y, et al. Pituitary dysfunction induced by immune checkpoint inhibitors is associated with better overall survival in both malignant melanoma and non-small cell lung carcinoma: a prospective study. Journal for ImmunoTherapy of Cancer 2020;8:e000779. doi:10.1136/ jitc-2020-000779

- Additional material is published online only. To view please visit the journal online (http://dx.doi.org/10.1136/jitc2020-000779).

Accepted 26 May 2020

Check for updates

(c) Author(s) (or their employer(s)) 2020. Re-use permitted under CC BY-NC. No commercial re-use. See rights and permissions. Published by BMJ.

For numbered affiliations see end of article.

\section{Correspondence to} Dr Shintaro Iwama; siwama@med.nagoya-u.ac.jp

Dr Hiroshi Arima;

arima105@med.nagoya-u.ac.jp

\section{ABSTRACT}

Background Several immune-related adverse events (irAEs) are reported to be associated with therapeutic efficacy of immune checkpoint inhibitors, yet whether pituitary dysfunction, a life-threatening irAE, affects overall survival (OS) in patients with malignancies is unclear. This prospective study examined the association of pituitary dysfunction (pituitary-irAE) with OS of patients with nonsmall cell lung carcinoma (NSCLC) or malignant melanoma (MM).

Methods A total of 174 patients (NSCLC, 108; MM, 66) treated with ipilimumab, nivolumab, pembrolizumab, or atezolizumab at Nagoya University Hospital were evaluated for OS and the development of pituitary-irAE. Kaplan-Meier curves of OS as a function of the development of pituitaryirAE were produced with the log-rank test as a primary endpoint.

Results Pituitary-irAE was observed in 16 patients (4 (3.7\%) with NSCLC, 12 (18.2\%) with MM) having two different disease types: hypophysitis with deficiency of multiple anterior pituitary hormones accompanied by pituitary enlargement, and isolated adrenocorticotropic hormone (ACTH) deficiency without pituitary enlargement. Among these patients, 6 developed pituitary-irAE while being treated with ipilimumab (6/25 patients $(24.0 \%)$ treated with ipilimumab) and 10 developed pituitaryirAE during treatment with nivolumab or pembrolizumab (10/167 (6.0\%)). All 16 patients had ACTH deficiency and were treated with physiological doses of hydrocortisone. The development of pituitary-irAE was associated with better OS in patients with NSCLC (not reached vs 441 (95\% Cl not calculated) days, $\mathrm{p}<0.05)$ and MM $(885(95 \%$ $\mathrm{Cl} 434$ to 1336) vs 298 (95\% Cl 84 to 512) days, $\mathrm{p}<0.05)$. Conclusions In our study cohort, the incidence of pituitary-irAE was higher than previously reported and the development of pituitary-irAE predicted better prognosis for both NSCLC and MM when patients were treated with physiological doses of hydrocortisone. Clinical trials registration UMIN000019024.

\section{BACKGROUND}

Immune checkpoint inhibitors (ICIs) including antibodies against anti-cytotoxic T-lymphocyte antigen 4 (CTLA-4) and anti-programmed cell death-1 (PD-1) have recently emerged as promising treatments for advanced malignancies. ${ }^{1}$ However, ICIs can cause adverse events, termed immune-related adverse events (irAEs), including pneumonitis, skin toxicities, colitis, and endocrine dysfunction. $^{23}$

ICIs were reported to have better antitumor efficacy in patients who developed some irAEs. ${ }^{4-9}$ In terms of the relationship of irAE to overall survival (OS), several studies reported that skin-irAE is associated with better OS in both patients with malignant melanoma (MM) and non-small cell lung carcinoma (NSCLC), ${ }^{4} 810 \quad 11$ whereas another study found that OS of patients with NSCLC was similar between those who developed skin-irAE and those who did not. ${ }^{7}$ The different outcomes of these previous clinical trials and retrospective studies could be due to the different study designs including evaluation methods and definition of irAEs. 


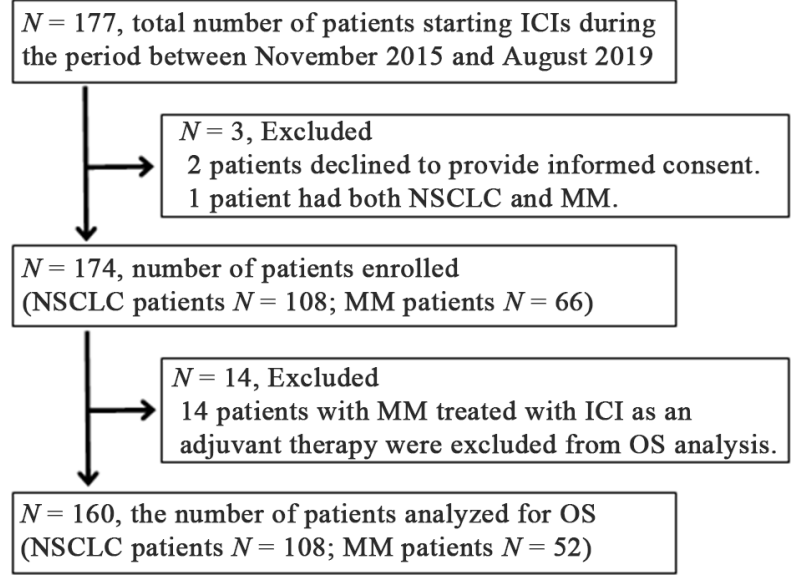

Figure 1 Enrollment of study subjects. ICl, immune checkpoint inhibitor; MM, malignant melanoma; NSCLC, nonsmall cell lung carcinoma; OS, overall survival.

Endocrine-irAEs comprise pituitary dysfunction, adrenal insufficiency, thyroid dysfunction, hypoparathyroidism, and type 1 diabetes mellitus (T1DM).$^{12}{ }^{13}$ Among them, thyroid-irAE induced by an anti-PD-1 antibody (aPD-1 Ab) was reported to be associated with better OS in patients with NSCLC in a phase I clinical trial ${ }^{14}$ and retrospective studies. ${ }^{51516}$ In contrast, thyroid-irAE induced by aPD-1 $\mathrm{Ab}$ was not associated with better OS of patients with $\mathrm{MM},{ }^{16}$ suggesting that the association of thyroid-irAE with improved outcomes varied among patients with NSCLC or MM. On the other hand, pituitary-irAE is almost always accompanied by adrenocorticotropic hormone (ACTH) deficiency, ${ }^{17}$ a life-threatening disorder, and a retrospective study reported that pituitary dysfunction induced by ipilimumab was associated with better OS in patients with MM. ${ }^{18}$ However, symptoms of ACTH deficiency, such as fatigue, appetite loss, and weight loss, frequently occur in patients with malignancies even if they do not develop pituitary-irAE. As such, pituitary-irAE could be overlooked when pituitary hormones are not measured regularly, and its incidence may not have been accurately reflected in previous studies.

To clarify the association of pituitary-irAE with OS in patients with NSCLC or MM, we performed a prospective study in which endocrine-irAEs induced by ICIs were set as a primary endpoint.

\section{METHODS \\ Patients}

To clarify the clinical features of endocrine-irAEs, we conducted a prospective study to analyze irAEs in patients treated with ICIs since November 2, 2015 (UMIN000019024). All patients with NSCLC or MM who started ICI treatment between November 2, 2015 and August 30, 2019 at Nagoya University Hospital were included in this study (figure 1). Patients who had more than two malignancies were excluded. Written informed consent was obtained from all patients. Ipilimumab was administered at $3 \mathrm{mg} / \mathrm{kg}$ every 3 weeks for 4 cycles. Nivolumab was administered at $3 \mathrm{mg} / \mathrm{kg}$ or $240 \mathrm{mg}$ every
2 weeks, with the exception of some patients with MM, who were treated with $2 \mathrm{mg} / \mathrm{kg}$ every 3 weeks. Pembrolizumab was administered at $2 \mathrm{mg} / \mathrm{kg}$ or $200 \mathrm{mg}$ every 3 weeks. Atezolizumab was administered at $1200 \mathrm{mg}$ every 3 weeks. All ICI treatment was continued until disease progressed, death, or unacceptable severe adverse events occurred, or if patients withdrew consent for treatment.

\section{Assessments}

To examine endocrine-irAEs, ACTH, cortisol, free T3 (FT3), free T4 (FT4), thyroid-stimulating hormone (TSH), and blood glucose levels were assessed at baseline and every 6 weeks after the first administration of ICI for 24 weeks as previously described..$^{19}$ After the initial 24 weeks, pituitary hormones were measured if clinically needed until the visits stopped. Each endocrine-irAE was defined according to the Japan Endocrine Society clinical guidelines for endocrine-irAEs. ${ }^{12}$ The cut-off for the diagnosis of ACTH deficiency was decreased peak serum cortisol value $(<18 \mu \mathrm{g} / \mathrm{dL})$ and impaired responses of ACTH $(<2$-fold of baseline) in corticotropin-releasing hormone loading tests (intravenous injection with $100 \mu \mathrm{g}$ human corticorelin), ${ }^{20}$ which were performed in the morning. The frequency of hyponatremia, defined as a serum sodium concentration $<135 \mathrm{mEq} / \mathrm{L}$, at the onset of pituitary-irAE was compared with that for patients who did not develop pituitary-irAE. In the latter group, the frequency was evaluated around the median time of onset of pituitary-irAE induced by each ICI (ipilimumab, 71 days; nivolumab, 171 days; pembrolizumab, 127 days). OS was determined for participants in this study until death from any cause. Patients with MM were excluded from the OS analysis if they were treated with ICIs as an adjuvant therapy (figure 1). Non-endocrine-irAEs, as reported in the guideline, ${ }^{13}$ were diagnosed by attending physicians and subjected to analysis if the grade was higher than 1. All irAEs were monitored and graded using CTCAE 5.0 criteria. Fifty-eight patients treated with nivolumab and 78 patients treated with pembrolizumab were included in our previous study that analyzed the incidence of thyroid dysfunction. ${ }^{1921}$

\section{Statistical analysis}

Continuous variables of patient characteristics (eg, age, days to diagnosis) are expressed as means $\pm \mathrm{SD}$ or median and IQR. Differences among continuous variables were tested for significance with the two-sample t-test. Values of nominal variables were compared using Fisher's exact test. OS was analyzed using the Kaplan-Meier method, expressed as median and 95\% CI and compared using a log-rank test. All statistical tests were two sided, and significance was defined as a $p$ value of $<0.05$. All statistical analyses were performed with IBM SPSS Statistics V.25.

\section{RESULTS}

\section{Patient characteristics}

A total of 174 patients with either NSCLC or MM who started ipilimumab, nivolumab, pembrolizumab, or atezolizumab therapy were enrolled in this study 
Table 1 Clinical characteristics and number of each irAE type among patients with NSCLC and MM

\begin{tabular}{|c|c|c|}
\hline & NSCLC & MM \\
\hline Characteristic & $n=108$ & $\mathrm{n}=66$ \\
\hline Age, years & $67 \pm 10$ & $69 \pm 12$ \\
\hline \multicolumn{3}{|l|}{ Sex } \\
\hline Female & 29 (26.9\%) & 27 (40.9\%) \\
\hline Male & $79(73.1 \%)$ & 39 (59.1\%) \\
\hline \multicolumn{3}{|l|}{ Drugs } \\
\hline Ipi & 0 & $24(36.4 \%)$ \\
\hline Niv & $57(52.8 \%)$ & $34(51.5 \%)$ \\
\hline Pem & $51(47.2 \%)$ & $30(45.5 \%)$ \\
\hline Ipi+Niv & 0 & $1(1.5 \%)$ \\
\hline Ate & $9(8.3 \%)$ & 0 \\
\hline History of prior ICl use & 0 & $12(18.2 \%)$ \\
\hline \multicolumn{3}{|l|}{ Treatment lines } \\
\hline Adjuvant & 0 & $14(21.2 \%)$ \\
\hline First line & 32 (29.6\%) & $33(50.0 \%)$ \\
\hline$\geq$ Second line & 76 (70.4\%) & $19(28.8 \%)$ \\
\hline Follow-up, days & $396 \pm 304$ & $343 \pm 319$ \\
\hline $\begin{array}{l}\text { Total no. of patients who } \\
\text { developed irAEs }\end{array}$ & 34 (31.5\%) & $38(57.6 \%)$ \\
\hline Pituitary-irAE & $4(3.7 \%)$ & $12(18.2 \%)$ \\
\hline Thyroid-irAE & $8(7.4 \%)$ & $11(16.7 \%)$ \\
\hline T1DM & $2(1.9 \%)$ & $1(1.5 \%)$ \\
\hline Lung-irAE & $13(12.0 \%)$ & $6(9.1 \%)$ \\
\hline Skin-irAE & $5(4.6 \%)$ & $4(6.1 \%)$ \\
\hline Gl-irAE & $4(3.7 \%)$ & $12(18.2 \%)$ \\
\hline Other & $3(2.8 \%)$ & $4(6.1 \%)$ \\
\hline
\end{tabular}

Data are mean \pm SD or $\mathrm{n}(\%)$.

Ate, atezolizumab; GI-irAE, gastrointestinal irAE; ICI, immune checkpoint inhibitor; Ipi, ipilimumab; irAE, immune-related adverse event; MM, malignant melanoma; Niv, nivolumab; NSCLC, non-small cell lung carcinoma; Pem, pembrolizumab; T1DM, type 1 diabetes mellitus.

(figure 1). Clinical variables for patients with NSCLC and $\mathrm{MM}$ are presented in table 1 . IrAEs occurred in $34 / 108$ patients $(31.5 \%)$ with NSCLC and $38 / 66$ patients (57.6\%) with MM (table 1). Endocrine-irAEs including pituitary-irAE, thyroid-irAE, and T1DM occurred in 13/108 (12.0\%) patients with NSCLC and 20/66 (30.3\%) patients with MM. The number of each type of irAE observed, including pituitary-irAE, thyroid-irAE, T1DM, interstitial pneumonitis (lung-irAE), skin toxicities (skinirAE), and gastrointestinal toxicities (GI-irAE), is shown in table 1 . No cases were treated with a reduced dose of ICI due to the development of irAEs.

\section{Pituitary dysfunction induced by ICls}

Pituitary-irAE occurred in 6/25 patients $(24.0 \%)$ during treatment with ipilimumab, $10 / 167$ patients $(6.0 \%)$ during treatment with nivolumab or pembrolizumab.

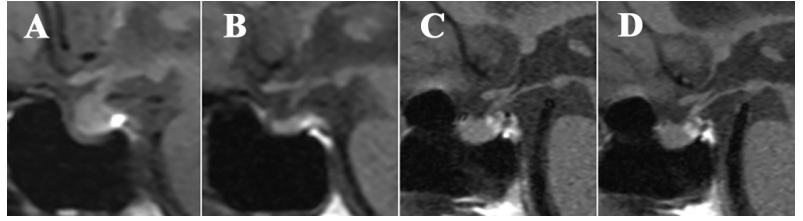

Figure $2 \mathrm{MRI}$ of the pituitary gland in a patient with pituitary immune-related adverse event (pituitary-irAE). Representative MRI for a patient (Ipi001) who developed hypophysitis shows that the pituitary gland was enlarged at pituitary-irAE onset (A) but had returned to normal size 6 months after the onset (B). Representative MRI for a patient (Niv096) who developed isolated adrenocorticotropic hormone deficiency show a normal-sized pituitary gland at pituitary-irAE onset $(C)$ and absence of changes at 6 months after onset (D).

Pituitary-irAE did not occur during treatment with atezolizumab (0/9). Pituitary-irAE induced by aPD-1 $\mathrm{Ab}$, nivolumab, or pembrolizumab was observed in $6 / 59$ patients with MM and 4/108 patients with NSCLC. There was no difference in the frequency of pituitary-irAE induced by aPD-1 Ab between patients with MM and those with NSCLC $(10.2 \%(6 / 59)$ in patients with MM vs $3.7 \%(4 / 108)$ in patients with NSCLC, $\mathrm{p}=0.168)$. Among the 16 patients who developed pituitary-irAE, three demonstrated clinical characteristics of hypophysitis such as pituitary enlargement and deficiencies in multiple anterior pituitary hormones including ACTH (online supplementary table S1). These three patients presented with headache as an initial symptom that could be caused by enlargement of the pituitary glands at irAE onset. A representative MRI showing an enlargement of the pituitary gland at hypophysitis onset is shown in figure 2A. This enlargement was completely resolved at 6 months after onset (figure 2B) and did not change during a 1-year follow-up (data not shown). The other 13 patients developed isolated ACTH deficiency (IAD), but none exhibited enlarged pituitary glands at IAD onset (online supplementary table S1 and figure 2C). Re-evaluation of pituitary gland size 6 months and/or 1 year after IAD onset for 11 of these patients showed no change in size for seven patients (representative MRI image, figure 2D) and a slightly decreased size for the other four patients. In terms of clinical characteristics of patients who developed hypophysitis and IAD, the usage rate of aCTLA- $4 \mathrm{Ab}$ and incidence of pituitary enlargement were significantly higher for patients who developed hypophysitis than those who had IAD (table 2). The time to diagnosis of pituitary-irAE after the first ICI administration was significantly shorter in patients with hypophysitis compared with those with IAD (table 2). There were no significant differences in the other clinical variables we examined (eg, malignancy type, age, sex, and history of prior immunotherapy) (table 2). ACTH deficiency was observed in all cases with pituitary-irAE (online supplementary table S2), and these patients were treated with physiological doses of hydrocortisone $(10-20 \mathrm{mg} /$ day $)$. ICI was re-administered with the same dose initially used after general conditions were stabilized by hormone replacement 
Table 2 Clinical characteristics of patients who developed pituitary-irAE

\begin{tabular}{|c|c|c|c|}
\hline & $\begin{array}{l}\text { Hypophysitis } \\
n=3\end{array}$ & $\begin{array}{l}\text { IAD } \\
n=13\end{array}$ & $P$ value \\
\hline Malignancy & & & 0.529 \\
\hline NSCLC & 0 & 4 (30.8\%) & \\
\hline MM & $3(100 \%)$ & $9(69.2 \%)$ & \\
\hline Age, years & $59 \pm 11$ & $67 \pm 10$ & 0.251 \\
\hline Sex & & & 0.509 \\
\hline Female & 0 & 5 (38.5\%) & \\
\hline Male & $3(100 \%)$ & $8(61.5 \%)$ & \\
\hline $\begin{array}{l}\text { History of prior } \\
\text { immunotherapy }\end{array}$ & 2 (66.7\%) & 3 (23.1\%) & 0.214 \\
\hline Latest ICI & & & 0.036 \\
\hline$a C T L A-4 \mathrm{Ab}$ & $3(100 \%)$ & $3(23.1 \%)$ & \\
\hline aPD-1 Ab & 0 & 10 (76.9\%) & \\
\hline $\begin{array}{l}\text { Days to diagnosis from } \\
\text { the first administration } \\
\text { of the latest drug }\end{array}$ & $56 \pm 27$ & $162 \pm 108$ & 0.008 \\
\hline $\begin{array}{l}\text { No. of cases with } \\
\text { enlarged pituitary by } \\
\text { MRI }\end{array}$ & $3(100 \%)$ & 0 & 0.002 \\
\hline
\end{tabular}

Data are $\mathrm{n}(\%)$ or mean \pm SD.

aCTLA-4 Ab, anti-cytotoxic T-lymphocyte antigen 4 antibodies; aPD-1 Ab, anti-programmed cell death-1 antibodies; IAD, isolated adrenocorticotropic hormone deficiency; ICl, immune checkpoint inhibitor; irAE, immune-related adverse event; MM, malignant melanoma; NSCLC, non-small cell lung carcinoma.

therapy. During the observation period of this study, no patients discontinued hormone replacement therapies (hydrocortisone and/or levothyroxine). The frequency of hyponatremia was significantly higher in patients who developed pituitary-irAE than those who did not $(6 / 16$ $(37.5 \%)$ vs $10 / 120(8.3 \%), \mathrm{p}<0.01)$.

\section{Thyroid dysfunction induced by ICls}

Thyroid-irAE occurred in 1/25 patients $(4.0 \%)$ during treatment with ipilimumab and in $18 / 167$ patients $(10.8 \%)$ during treatment with nivolumab or pembrolizumab. The incidence of thyroid-irAE tended to be higher in patients treated with $\mathrm{APD}-1 \mathrm{Ab}$ than those who received aCTLA-4 Ab, although the difference was not significant $(18 / 167(10.8 \%)$ vs $1 / 25(4.0 \%), p=0.477)$. The number of patients who developed destructive thyroiditis, hypothyroidism, and hyperthyroidism was 13,5 , and 1 , respectively. All five patients who developed hypothyroidism and 12/13 who developed destructive thyroiditis required hormone therapy with levothyroxine.

\section{Patients who developed any irAE had longer OS than those without irAEs}

OS was significantly longer for both patients with NSCLC and $\mathrm{MM}$ who developed any irAE than those without irAEs (NSCLC: not reached vs 378 (95\% CI 220 to 536) days, $\mathrm{p}<0.01$, figure $3 \mathrm{~A}$, online supplementary table $\mathrm{S} 3$; MM: 534 (95\% CI 245 to 823 ) vs 189 (95\% CI 52 to 326) days, $\mathrm{p}<0.05$, figure $3 \mathrm{~B}$, online supplementary table S3). To examine the contribution of endocrine-irAEs to increased OS, 13 patients with NSCLC and 14 patients with MM who developed endocrine-irAEs were included in OS analysis. OS was significantly longer for patients with NSCLC who developed endocrine-irAEs than those who did not (not reached vs 401 days (95\% CI 240 to 562 ), $\mathrm{p}<0.01$, figure 3C, online supplementary table S3). In patients with MM, there were no significant differences in OS between patients who developed endocrine-irAEs and those who did not (649 days (95\% CI 367 to 931) vs 342 days (95\% CI 131 to 553), $\mathrm{p}=0.107$, figure $3 \mathrm{D}$, online supplementary table S3).

\section{Pituitary-irAE was associated with prolonged OS in patients with NSCLC or MM}

Four patients with NSCLC and ninepatients with MM with pituitary-irAE were included in the analysis of OS. OS was significantly longer for both patients with NSCLC and MM who developed pituitary-irAE than those who did not (NSCLC: not reached vs 441 days $(95 \%$ CI not calculated), $\mathrm{p}<0.05$, figure $3 \mathrm{E}$, online supplementary table S3; MM: 885 days (95\% CI 434 to 1336) vs 298 says (95\% CI 84 to 512$), \mathrm{p}<0.05$, figure $3 \mathrm{~F}$, online supplementary table S3). There were no significant differences between patients with NSCLC (online supplementary table S4) or MM (online supplementary table S5) who developed pituitaryirAE and those who did not in terms of sex, drugs used, prior history of other ICIs, and treatment lines, although the mean ages were significantly younger and the ipilimumab usage rate was significantly higher for patients with MM who developed pituitary-irAE compared with those who did not (online supplementary table S5). The analysis of OS included patients treated with ICI monotherapy as well as sequential use of several ICIs because the number of patients who were treated with only one ICI during the study period was too small (online supplementary table S6). There were no significant differences in the usage rate of multiple ICIs between patients who developed pituitaryirAE for patients with either NSCLC $(0 / 4$ with pituitary-irAE vs $8 / 104$ without pituitary-irAE. $\mathrm{p}=1.000)$ or MM $(5 / 9$ with pituitary-irAE vs $20 / 43$ without pituitary-irAE. $\mathrm{p}=0.722$ ). There were no differences in the number of cycles of ICI between patients who developed pituitary-irAE and those who did not, except for patients with MM treated with nivolumab (online supplementary tables $\mathrm{S} 7$ and S8). In this group, the number of cycles was significantly higher in the pituitary-irAE group (online supplementary table S8). The time of follow-up was significantly longer for the patients with NSCLC and MM who developed pituitary-irAE than those who did not (online supplementary tables S7 and S8).

\section{Thyroid-irAE was associated with prolonged OS in patients with NSCLC, but not MM}

OS was significantly longer for patients with NSCLC who developed thyroid-irAE than those who did not (not reached vs 441 days (95\% CI 317 to 565), p $<0.05$, figure $3 \mathrm{G}$, online supplementary table S3). In contrast, 
A

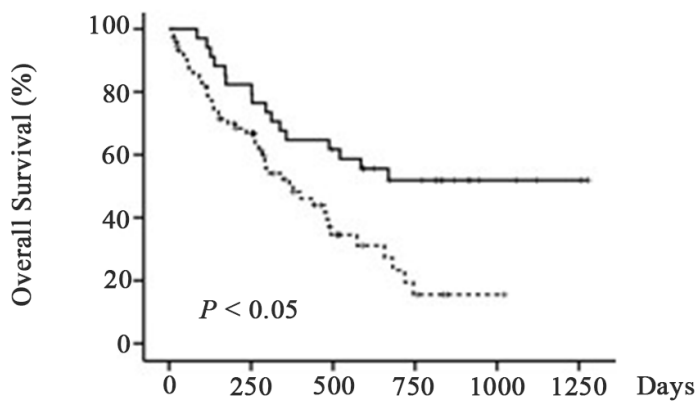

No. at risk

Any-irAEs 34

No irAEs 74

C

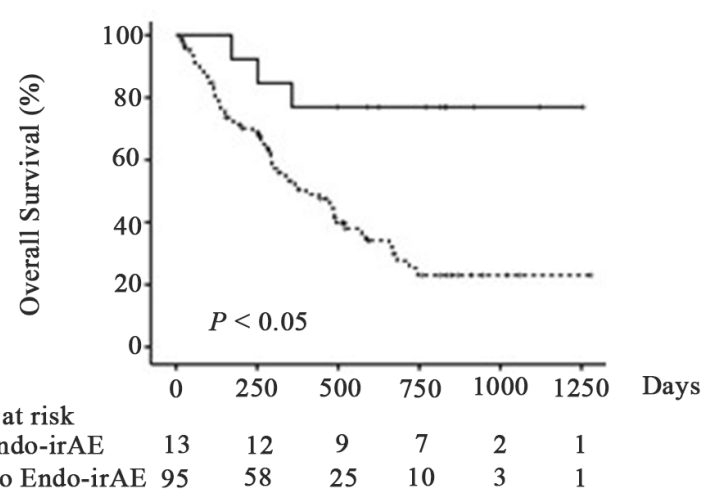

$\mathbf{E}$

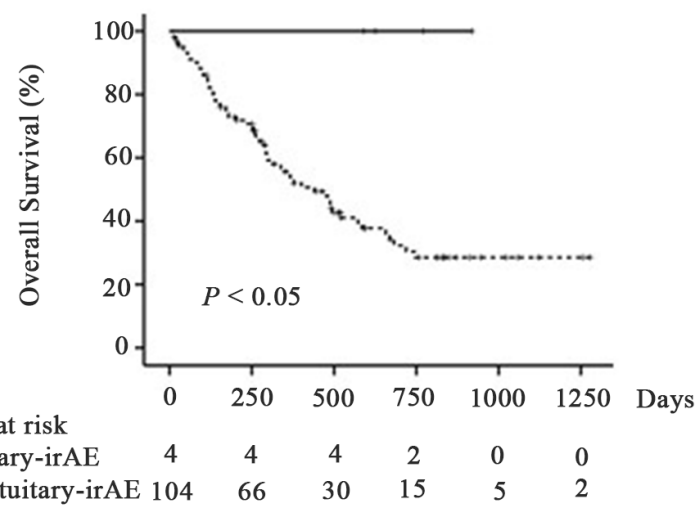

G

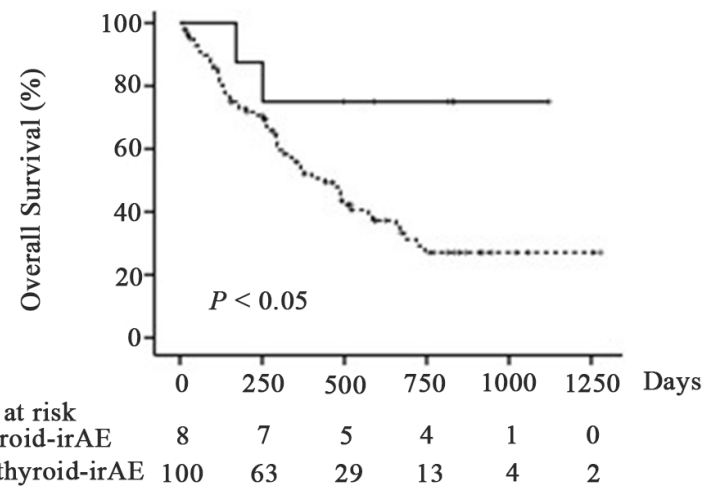

B

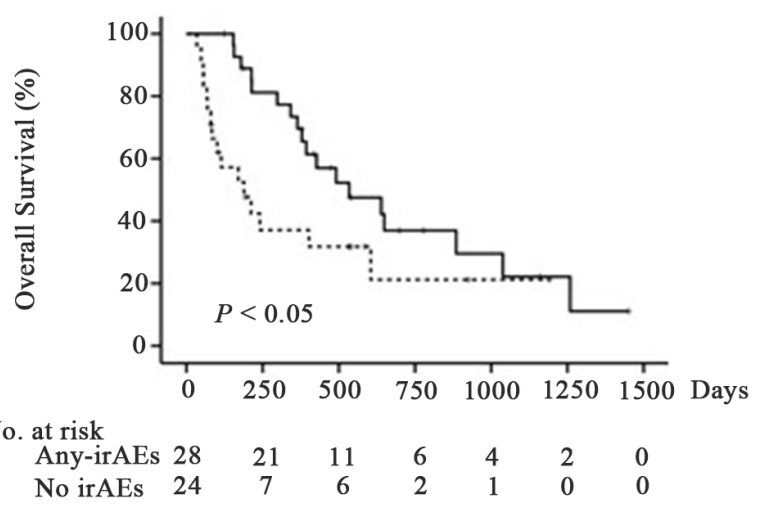

D

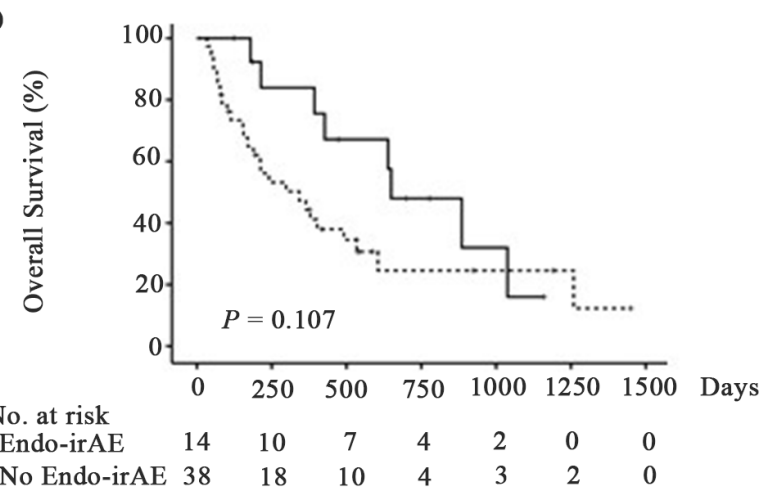

$\mathbf{F}$

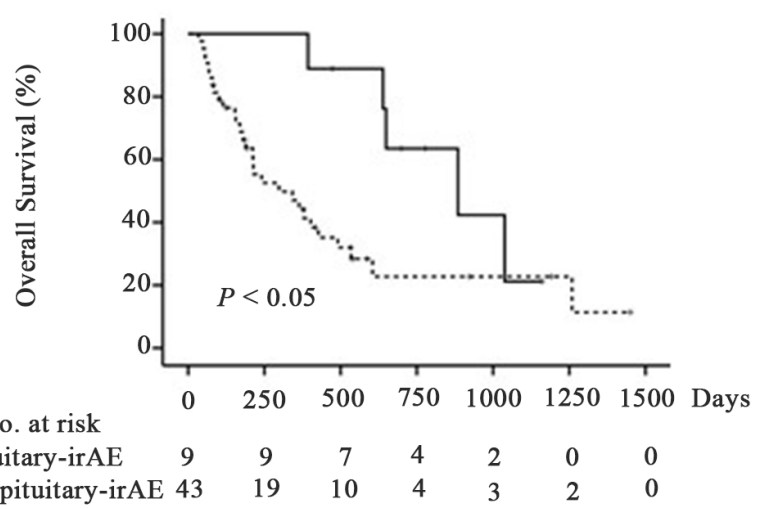

$\mathbf{H}$

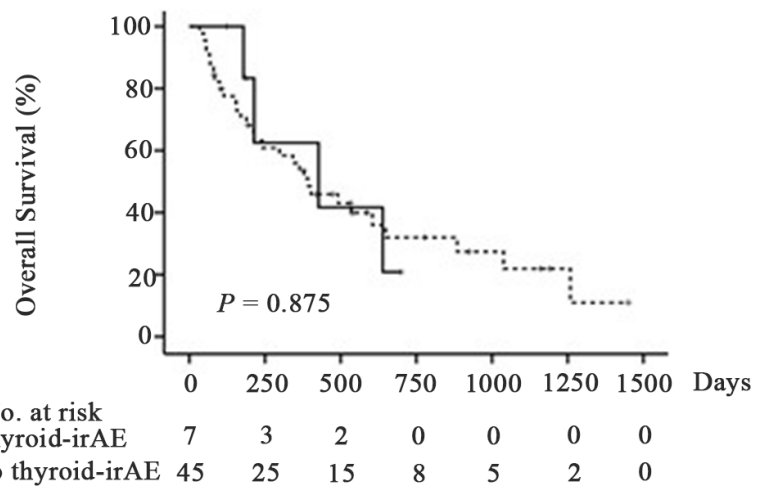

Figure 3 Overall survival (OS) after initiation of immune checkpoint inhibitor treatment in patients with and without immunerelated adverse events (irAEs). OS of patients with non-small cell lung carcinoma (NSCLC) (A, C, E, G) or malignant melanoma (MM) (B, D, F, H). Solid and dashed lines indicate OS of patients who did and did not develop each rAE, respectively. The OS was significantly prolonged for patients who developed any irAEs than those who did not for both patients with NSCLC and MM (A, B). The OS was significantly prolonged for patients who developed endocrine-irAEs than those who did not for patients with NSCLC (C) but not patients with MM (D). Among patients with NSCLC and those with MM, the OS was significantly prolonged for patients who developed pituitary-irAE than those who did not (E, F). The OS was significantly prolonged for patients who developed thyroid-irAE than those who did not for patients with NSCLC (G) but not patients with MM $(H)$.

Kobayashi T, et al. J Immunother Cancer 2020;8:e000779. doi:10.1136/jitc-2020-000779

5 
patients with MM who developed thyroid-irAE and those who did not had similar OS (427 days (95\% CI 0 to 871) vs 393 days (95\% CI 219 to 567), p=0.875, figure $3 \mathrm{H}$, online supplementary table S3). There were no significant differences between patients with NSCLC (online supplementary table S9) or MM (online supplementary table S10) who developed thyroid-irAE and those who did not for other clinical factors including sex, drugs used, prior history of other ICIs, and treatment lines. Moreover, there were no significant differences in the usage rate of multiple ICIs between patients who developed thyroidirAE and those who did not among patients with NSCLC (0/8 with thyroid-irAE vs $8 / 100$ without thyroid-irAE; $\mathrm{p}=1.000)$ or MM ( $2 / 7$ with thyroid-irAE vs $24 / 45$ without thyroid-irAE; $\mathrm{p}=0.419)$.

\section{OS was not associated with other types of irAE in patients with NSCLC or MM}

Although patients who had any irAE showed longer OS than those without irAEs, the development of nonendocrine-irAEs was not associated with longer OS in patients with either NSCLC or MM (NSCLC: 585 days (95\% CI 321 to 849 ) vs 441 days (95\% CI 297 to 585), $\mathrm{p}=0.247$, online supplementary figure $1 \mathrm{~A}$, table S3; MM: 534 days (95\% CI 268 to 800 ) vs 241 days (95\% CI 0 to 523), $\mathrm{p}=0.268$, online supplementary figure $1 \mathrm{~B}$, table S3). As for each non-endocrine-irAE, there were no differences in OS between patients who developed lung-irAE, skin-irAE, or GI-irAE and those who did not for patients with NSCLC (lung-irAE, 488 days (95\% CI 167 to 809) vs 474 days (95\% CI 334 to 614 ), $\mathrm{p}=0.710$; skin-irAE, 669 days (95\% CI 438 to 900$)$ vs 441 days (95\% CI 310 to 572 ), $\mathrm{p}=0.550$; GI-irAE, not reached vs 481 days (95\% CI 351 to $597), \mathrm{p}=0.731$ ) or MM (lung-irAE, 1259 days $(95 \%$ CI 0 to 2655 ) vs 393 days ( $95 \%$ CI 237 to 549 ), $\mathrm{p}=0.202$; skinirAE, 649 days (95\% CI not calculated) vs 379 (95\% CI 252 to 506), $\mathrm{p}=0.560$; GI-irAE, 491 days $(95 \%$ CI 152 to 830 ) vs 393 days (95\% CI 174 to 612), $\mathrm{p}=0.581$ ) (online supplementary figure $1 \mathrm{C}-\mathrm{H}$, table $\mathrm{S} 3$ ). For skin-irAEs, exanthema and erythema, but not vitiligo, were observed in this study.

\section{DISCUSSION}

This prospective study, in which all patients with pituitaryirAE were treated with physiological doses of hydrocortisone, revealed that pituitary-irAE was associated with prolonged OS in patients with MM or NSCLC. Furthermore, our study confirmed the findings of previous retrospective studies and a phase I trial that thyroid-irAE was associated with prolonged OS in patients with NSCLC but not those with MM. Thus, the outcome of pituitaryirAEs and thyroid-irAEs in terms of OS differed between NSCLC and MM.

The results of this prospective study clearly demonstrated that the incidence of pituitary-irAE was higher (24.0\% and $6.0 \%$ for patients treated with aCTLA- $4 \mathrm{Ab}$ and aPD-1 Ab, respectively) than previously reported. ${ }^{17}$ 22-24
In a retrospective analysis of a medical record database, Faje et al reported that the incidence of pituitary dysfunction induced by aCTLA- $4 \mathrm{Ab}$ and aPD- $1 \mathrm{Ab}$ was $13.6 \%$ or $0.5 \%$, respectively. ${ }^{25}$ Pituitary-irAE could have been overlooked in that study because most symptoms associated with pituitary-irAE such as fatigue, appetite loss, and weight loss also occur in patients with malignancies. One strength of the present prospective study is that pituitaryirAE was far more likely to be detected because of the scheduled endocrine evaluation during the fixed period regardless of whether ICIs were continued. In addition, this study has less selection bias than clinical trials because all patients treated with ICIs in our hospital were prospectively included. Thus, the incidence of pituitaryirAE observed in this study is more likely to reflect that which occurs in a real-world clinical practice.

Concerning the association of pituitary-irAE with OS, only one retrospective study that analyzed a clinical record database reported an association of pituitary dysfunction induced by a CTLA-4 Ab (ipilimumab) with prolonged $\mathrm{OS}$ in patients with $\mathrm{MM}^{18}$ and no prior studies have shown an association of pituitary-irAE with OS in patients with NSCLC. It is also unclear whether pituitary dysfunction induced by aPD-1 Ab is associated with OS. This study analyzed the association of OS with pituitary dysfunction induced by aCTLA-4 Ab or aPD-1 Ab, and clarified that pituitary-irAE is associated with prolonged OS in patients with MM and also those with NSCLC. Thus, pituitary-irAE could be a potential biomarker to predict better outcome of ICI treatments for these types of malignancies, although it sometimes develops several months after the initial administration of ICIs.

Given the contribution of pituitary-irAE to better OS, it is important to understand its clinical characteristics, how to manage them appropriately, and why pituitaryirAE is associated with prolonged survival. This study clearly demonstrated that ICI can induce two types of pituitary dysfunction: hypophysitis, associated with deficiencies in multiple pituitary hormones accompanied by pituitary enlargement, and IAD without pituitary enlargement. Our data also showed that (1) ACTH deficiency was observed in all cases, (2) IAD can be induced by either aCTLA-4 Ab or aPD-1 Ab, and (3) hypophysitis was induced only by aCTLA- $4 \mathrm{Ab}$.

The mechanisms underlying the association of pituitary-irAE with better outcome of ICI treatments are still unknown. The development of vitiligo was associated with better tumor response in patients with $\mathrm{MM},{ }^{10}$ suggesting that melanoma cells and normal melanocytes may have shared antigens. It would be interesting to explore whether there are shared antigens between pituitary cells and MM or NSCLC cells to determine if they are involved in development of pituitary-irAE. Previous studies proposed that complement activation following aCTLA-4 Ab binding to CTLA-4 expressed on pituitary cells is involved in pathogenesis of hypophysitis induced by aCTLA- $4 \mathrm{Ab}$ in mice ${ }^{26}$ and humans. ${ }^{17}$ Given that aCTLA-4 Ab is an IgG1 and aPD-1 Ab is an IgG4 that 
cannot activate complement, the mechanism underlying pituitary-irAEs likely differs among ICIs.

Determining effective treatment strategies for pituitary dysfunction induced by ICIs is critical. Although the effect of high-dose glucocorticoids to treat pituitaryirAE has not been fully examined, retrospective studies reported that such doses did not affect recovery of pituitary function ${ }^{27}$ and instead shortened OS. ${ }^{18}$ In this study, all patients who developed pituitary-irAE were treated with physiological doses of hydrocortisone. In addition, all patients who developed pituitary-irAE were re-administered ICIs after their general conditions were stabilized by hormone treatment. Although pituitaryirAE can be life threatening, ${ }^{28}{ }^{29}$ this study demonstrated the association of pituitary-irAE with better OS when patients are treated with physiological doses of hydrocortisone.

Although hyponatremia can accompany adrenal insufficiency, some patients with cancer also often develop hyponatremia ${ }^{30}$ due to inappropriate anti-diuretic hormone secretion, ${ }^{31}$ gastrointestinal or renal losses and/or diminished sodium intake, use of diuretics, hypervolemic state, renal failure, or intake of hypotonic fluids. ${ }^{32}{ }^{33}$ In this study, we first demonstrated that the frequency of hyponatremia was significantly higher in the patients who developed pituitary-irAE compared with those who did not. These data suggest that ACTH and cortisol levels in the blood should be measured to detect development of pituitary-irAE when hyponatremia is observed in patients treated with ICIs.

Previous studies reported an association between better OS with irAEs other than endocrine-irAEs, including skin-irAE or lung-irAE. 781011 We clearly demonstrated the association of pituitary-irAE with better ICI treatment outcomes, yet no irAE type other than endocrine-irAEs was associated with better OS. Endocrine-irAEs were definitely determined as primary outcomes according to Japan Endocrine Society clinical guidelines, ${ }^{12}$ whereas other irAEs were diagnosed by attending physicians in practice, which may have affected the results of this study.

This study has some limitations. First, analysis of OS for each ICI regimen did not yield statistically significant results likely due to small sample sizes. We therefore included both patients treated with ICI monotherapy as well as those treated with several ICIs sequentially in the analyses. Second, to calculate the incidence of pituitary dysfunction, we defined the drug that was in use at onset of pituitary dysfunction to be the causative drug, and we cannot exclude the possibility that a previously used ICI may have affected the development of irAEs. Third, there may be a bias in that pituitary-irAE can be observed in patients who survived for a longer time. Actually, some patients showed late onset of pituitary-irAE in this study. Additional studies that include landmark analyses for larger study populations are needed to address these limitations.

\section{CONCLUSIONS}

Our prospective study clarified the exact incidence of pituitary-irAE in a real-world clinical practice and the association of pituitary-irAE accompanied by ACTH deficiency with a better outcome of ICI treatments in both NSCLC and MM when patients were treated with physiological doses of hydrocortisone. Our findings indicate that we should measure pituitary hormones when pituitary-irAE is suspected based on hyponatremia, so that ACTH deficiency in patients treated with ICI will not be overlooked.

\section{Author affiliations}

${ }^{1}$ Department of Endocrinology and Diabetes, Nagoya University Graduate School of Medicine, Nagoya, Japan

${ }^{2}$ Department of CKD Initiatives Internal Medicine, Nagoya University Graduate School of Medicine, Nagoya, Japan

${ }^{3}$ Research Center of Health, Physical Fitness and Sports, Nagoya University, Nagoya, Japan

${ }^{4}$ Department of Dermatology, Nagoya University Graduate School of Medicine,

Nagoya, Japan

${ }^{5}$ Department of Respiratory Medicine, Nagoya University Graduate School of

Medicine, Nagoya, Japan

${ }^{6}$ Center for Advanced Medicine and Clinical Research, Nagoya University Hospital, Nagoya, Japan

${ }^{7}$ Department of Otorhinolaryngology, Nagoya University Graduate School of

Medicine, Nagoya, Japan

${ }^{8}$ Department of Oral and Maxillofacial Surgery, Nagoya University Graduate School of Medicine, Nagoya, Japan

${ }^{9}$ Department of Clinical Oncology and Chemotherapy, Nagoya University Hospital, Nagoya, Japan

Contributors SI designed the study. TK and SI performed the clinical study. TK, $\mathrm{SI}$, and $\mathrm{HA}$ analyzed the data. All authors treated the patients who were enrolled, collected and discussed the data. TK, SI, and HA wrote the manuscript. All authors were involved in revising the manuscript.

Funding The authors have not declared a specific grant for this research from any funding agency in the public, commercial or not-for-profit sectors.

Competing interests $\mathrm{SI}$ receives personal fees from Ono Pharmaceutical Company, Bristol-Myers Squibb, MSD K.K., and Chugai Pharmaceutical Co., Ltd. T0 receives personal fees from MSD K.K. HT receives grants from MSD K.K. YI receives grants from Sanwa Kagaku Kenkyusho, Kowa Pharmaceutical, MSD K.K., Dainippon Sumitomo, Kyowa Kirin Co., Ltd., Chugai Pharmaceutical Co., Ltd., Boehringer Ingelheim, Nihon Medi-Physics Co., Ltd., and personal fees from Astellas Pharma, Daiichi Sankyo, and Ono Pharmaceutical Company. KY receives personal fees from Ono Pharmaceutical Company, MSD K.K., and Bristol-Myers Squibb. TH received personal fees from Chugai Pharmaceutical Co., Ltd., Ono Pharmaceutical Company, and Bristol-Myers Squibb while the study was being conducted. TH also received grants from Boehringer Ingelheim, Taiho Pharmaceutical Co., Ltd., and personal fees from Boehringer Ingelheim outside the submitted work. MMS receives personal fees from Ono Pharmaceutical Company, Bristol-Myers Squibb, MSD K.K., and Chugai Pharmaceutical Co., Ltd. MA receives grants from Kyowa Kirin Co., Ltd. $Y A$ receives grants and personal fees from Ono Pharmaceutical Company, Chugai Pharmaceutical Co., Ltd., and Taiho Pharmaceutical Co. Ltd. as well as personal fees from Bristol-Myers Squibb. MA receives grants and personal fees from Ono Pharmaceutical Company, MSD K.K., and personal fees from Bristol-Myers Squibb. YH receives grants and personal fees from Ono Pharmaceutical Company, Chugai Pharmaceutical Co., Ltd., Eli Lilly, Boehringer Ingelheim, Bristol-Myers Squibb, MSD K.K., and Pfizer; grants from Novartis and Taiho Pharmaceutical Co., Ltd., and personal fees from Astra Zeneca. HA receives grants from Ono Pharmaceutical Company, MSD K.K., Chugai Pharmaceutical Co. Ltd., and personal fees from Ono Pharmaceutical Company, Bristol-Myers Squibb, and MSD K.K.

Patient consent for publication Not required.

Ethics approval This study was approved by the Ethical Committee of Nagoya University Hospital, No. 2015-0273. Written informed consent was obtained from all patients. 
Provenance and peer review Not commissioned; externally peer reviewed.

Data availability statement Data are available on reasonable request.

Open access This is an open access article distributed in accordance with the Creative Commons Attribution Non Commercial (CC BY-NC 4.0) license, which permits others to distribute, remix, adapt, build upon this work non-commercially, and license their derivative works on different terms, provided the original work is properly cited, appropriate credit is given, any changes made indicated, and the use is non-commercial. See http://creativecommons.org/licenses/by-nc/4.0/.

\section{ORCID iD}

Shintaro Iwama http://orcid.org/0000-0002-3281-0337

\section{REFERENCES}

1 Postow MA, Callahan MK, Wolchok JD. Immune checkpoint blockade in cancer therapy. JCO 2015;33:1974-82.

2 Scott LJ. Nivolumab: a review in advanced melanoma. Drugs 2015;75:1413-24.

3 Graziani G, Tentori L, Navarra P. Ipilimumab: a novel immunostimulatory monoclonal antibody for the treatment of cancer. Pharmacol Res 2012;65:9-22.

4 Freeman-Keller M, Kim Y, Cronin $\mathrm{H}$, et al. Nivolumab in resected and unresectable metastatic melanoma: characteristics of immunerelated adverse events and association with outcomes. Clin Cancer Res 2016;22:886-94.

5 Grangeon M, Tomasini P, Chaleat S, et al. Association between immune-related adverse events and efficacy of immune checkpoint inhibitors in non-small-cell lung cancer. Clin Lung Cancer 2019;20:201-7.

6 Indini A, Di Guardo L, Cimminiello C, et al. Immune-related adverse events correlate with improved survival in patients undergoing antiPD1 immunotherapy for metastatic melanoma. J Cancer Res Clin Oncol 2019;145:511-21.

7 Ricciuti B, Genova C, De Giglio A, et al. Impact of immune-related adverse events on survival in patients with advanced non-small cell lung cancer treated with nivolumab: long-term outcomes from a multi-institutional analysis. J Cancer Res Clin Oncol 2019;145:479-85.

8 Haratani K, Hayashi H, Chiba Y, et al. Association of immune-related adverse events with nivolumab efficacy in non-small-cell lung cancer JAMA Oncol 2018;4:374-8.

9 Okada N, Kawazoe H, Takechi K, et al. Association between immune-related adverse events and clinical efficacy in patients with melanoma treated with nivolumab: a multicenter retrospective study. Clin Ther 2019;41:59-67.

10 Teulings H-E, Limpens J, Jansen SN, et al. Vitiligo-like depigmentation in patients with stage III-IV melanoma receiving immunotherapy and its association with survival: a systematic review and meta-analysis. J Clin Oncol 2015;33:773-81.

11 Fujisawa Y, Yoshino K, Otsuka A, et al. Retrospective study of advanced melanoma patients treated with ipilimumab after nivolumab: analysis of 60 Japanese patients. J Dermatol Sci 2018;89:60-6.

12 Arima $\mathrm{H}$, Iwama $\mathrm{S}$, Inaba $\mathrm{H}$, et al. Management of immune-related adverse events in endocrine organs induced by immune checkpoint inhibitors: clinical guidelines of the Japan Endocrine Society. Endocr J 2019;66:581-6.

13 Brahmer JR, Lacchetti C, Schneider BJ, et al. Management of immune-related adverse events in patients treated with immune checkpoint inhibitor therapy: American Society of Clinical Oncology clinical practice guideline. J Clin Oncol 2018;36:1714-68.
14 Osorio JC, Ni A, Chaft JE, et al. Antibody-mediated thyroid dysfunction during T-cell checkpoint blockade in patients with nonsmall-cell lung cancer. Ann Oncol 2017;28:583-9.

$15 \mathrm{Kim} \mathrm{HI}$, Kim M, Lee S-H, et al. Development of thyroid dysfunction is associated with clinical response to PD-1 blockade treatment in patients with advanced non-small cell lung cancer. Oncoimmunology 2017;7:e1375642.

16 Yamauchi I, Yasoda A, Matsumoto S, et al. Incidence, features, and prognosis of immune-related adverse events involving the thyroid gland induced by nivolumab. PLoS One 2019;14:e0216954.

17 Caturegli P, Di Dalmazi G, Lombardi M, et al. Hypophysitis secondary to cytotoxic T-lymphocyte-associated protein 4 blockade: insights into pathogenesis from an autopsy series. Am J Pathol 2016;186:3225-35.

18 Faje AT, Lawrence D, Flaherty K, et al. High-dose glucocorticoids for the treatment of ipilimumab-induced hypophysitis is associated with reduced survival in patients with melanoma. Cancer 2018;124:3706-14.

19 Kobayashi T, Iwama S, Yasuda Y, et al. Patients with antithyroid antibodies are prone to develop destructive thyroiditis by nivolumab: a prospective study. J Endocr Soc 2018;2:241-51.

20 Yanase T, Tajima T, Katabami T, et al. Diagnosis and treatment of adrenal insufficiency including adrenal crisis: a Japan Endocrine Society clinical practice guideline [Opinion]. Endocr $J$ 2016;63:765-84

21 Okada N, Iwama S, Okuji T, et al. Anti-thyroid antibodies and thyroid echo pattern at baseline as risk factors for thyroid dysfunction induced by anti-programmed cell death-1 antibodies: a prospective study. Br J Cancer. In Press 2020;122:771-7.

22 Faje A. Immunotherapy and hypophysitis: clinical presentation, treatment, and biologic insights. Pituitary 2016;19:82-92.

23 Corsello SM, Barnabei A, Marchetti P, et al. Endocrine side effects induced by immune checkpoint inhibitors. J Clin Endocrinol Metab 2013;98:1361-75.

24 González-Rodríguez E, Rodríguez-Abreu D, Spanish Group for Cancer Immuno-Biotherapy (GETICA). Immune checkpoint inhibitors: review and management of endocrine adverse events. Oncologist 2016;21:804-16.

25 Faje A, Reynolds K, Zubiri L, et al. Hypophysitis secondary to nivolumab and pembrolizumab is a clinical entity distinct from ipilimumab-associated hypophysitis. Eur J Endocrinol 2019;181:211-9.

26 Iwama S, De Remigis A, Callahan MK, et al. Pituitary expression of CTLA-4 mediates hypophysitis secondary to administration of CTLA4 blocking antibody. Sci Transl Med 2014;6:230ra245.

27 Min L, Hodi FS, Giobbie-Hurder A, et al. Systemic high-dose corticosteroid treatment does not improve the outcome of ipilimumab-related hypophysitis: a retrospective cohort study. Clin Cancer Res 2015;21:749-55.

28 Arlt W, Allolio B. Adrenal insufficiency. Lancet 2003;361:1881-93.

29 Bergthorsdottir R, Leonsson-Zachrisson M, Odén A, et al. Premature mortality in patients with Addison's disease: a population-based study. J Clin Endocrinol Metab 2006;91:4849-53.

30 Berardi R, Antonuzzo A, Blasi L, et al. Practical issues for the management of hyponatremia in oncology. Endocrine 2018;61:158-64.

31 Raftopoulos H. Diagnosis and management of hyponatremia in cancer patients. Support Care Cancer 2007;15:1341-7.

32 Berghmans T, Paesmans M, Body JJ. A prospective study on hyponatraemia in medical cancer patients: epidemiology, aetiology and differential diagnosis. Support Care Cancer 2000;8:192-7.

33 Onitilo AA, Kio E, Doi SAR. Tumor-related hyponatremia. Clin Med Res 2007;5:228-37. 